\title{
A note on the search for 'optimal' shapes of arch dams
}

\author{
M. Fanelli ${ }^{1} \&$ A. Fanelli ${ }^{2}$ \\ ${ }^{1}$ Private Consultant, Former Director of the Centre for Hydraulic \& \\ Structural Research of ENEL, Italy \\ ${ }^{2}$ Private Consultant, Italy
}

\begin{abstract}
The 'shape resistance' of an arch dam affords a concrete volume substantially smaller than that of a gravity dam of equal height, at the expense of more stringent requirements for concrete quality, formwork, foundation treatments, geometric tracing and erection constraints. Minimisation of the concrete volume or, more generally, of total costs is pursued by 'optimising' the shape under a set of constraints. This requires defining a 'structural model', a set of 'shape parameters', the 'constraints' under which optimisation is to be achieved, the 'design external load(s)', an 'objective function' and an 'algorithmic procedure'. Usually the number of shape parameters ranges from a dozen to 40 or more, to leave wide freedom of adaptation of the final shape to loads and constraints, and a unique distribution of design loads is assumed; hence heavy analyticalnumerical procedures leading to a unique 'optimal' shape. As the dam is called to withstand a large variety of external actions, the shape thus obtained cannot be 'optimal' for all cases; therefore the rationale for complex optimisation models may be questioned. The Authors claim that the number of shape parameters can be kept very small (from three to a few units) by adopting a simplified model complying from the start with the main constraints. This claim is supported by past experience: the shapes of arch dams designed in this way were found, indeed, to be very close to those obtained by more 'rigorous' approaches.
\end{abstract}

Keywords: arch dams, shape optimisation, shape parameters, constraints, simplified models. 


\section{Introduction}

State of the art does not allow 'direct' design of an arch dam. Experience, trialand-error procedures, algorithmic 'optimisation' methods are the tools currently available in order to define a structurally adequate shape avoiding unnecessary costs. In principle only an infinite number of geometrical parameters could accurately define a shape which is totally unknown to start with. Even if in actual practice a search for such an extreme accuracy is not warranted for, in most of the literature a large number of geometrical parameters (between a dozen and several tens) is currently deemed necessary to leave adequate 'freedom' to the search strategy. The 'objective function' to be minimized (under the given constraints) is usually defined as the overall cost of erection, of which the main components are proportional to i) the volume of concrete and ii) the surface and volume of the excavations and foundation works (sometimes only i) is considered). The constraints under which 'shape optimisation' is to be sought relate mainly to geometry (adaptation to local topography/geology), to the building schedule (e.g. the choice to erect the individual monoliths independently from each other, i.e. avoiding the necessity of coordinated pouring and progressive joint grouting.) and to structural adequacy, i.e. the capacity to withstand a given set of loads without 'failure' (for the definition of 'failure' see the ICOLD glossary). As to the latter, 'admissible stress' resistance criteria are assumed; but the constrained stresses are not explicit functions of the shape parameters, and to find the minimum of a function of a large number of variables under implicit constraints turns out to be a computationally expensive task (FE stress analysis is currently used). Moreover, the 'precision' of the solution pursued by such a search can be delusory, insofar as the purported 'optimal' shape is obtained in relation to a definite (unique) set of loads, whereas the 'real' dam is called to face a large variety of different load scenarios. Simplified optimisation methods preserving the essential features of the problem and allowing for judicious intervention of the designer's experience may therefore be proposed (if proven valid in practice) as more expeditious approaches. The present note suggests that a pared-down model based on suitably chosen variables might avoid FE computing and reduce the degrees of freedom of the search to only three/four.

\section{Generalities}

Arch dam structural analysis encompasses two main classes of problems: i) analysis of existing (or anyway pre-defined) structures and ii) design of dams still to be built. The 'brute force' approach to the second problem would proceed through a 'trial and error' procedure. To wit, a large number of 'compatible' geometric shapes (i.e. shapes compatible with the local topography/geology.) (generated either by a systematic scanning of the possibilities or by the designer's intuition and experience) would be analysed by the methods used for the first class of problems (i); a screening of the results would then identify the solution(s) compatible with the constraints and presenting as little costs as 
possible. While there is nothing wrong in this way of proceeding, more satisfactory lines of approach are devised, i.e. ones that embody greater objectivity (as compared with the experienced engineer's intuitive approach) avoiding at the same time excessive computational costs (as compared with the brute-force trial-and-error approach). The choice among the many options for such methodologies should be guided by the criterion that a cunning selection of the main variables is the best premise to identifying an effective mathematical model of the problem in hand (J.H. POINCARE'). The approach herein presented starts from the classical RITTER idea of splitting the design load into two fractions: one carried by the bending stiffness of the vertical cantilevers and the remaining part carried by the axial stiffness of the horizontal arches. The stress distribution caused by dead weight is considered apart, under the assumption that the individual monoliths are so designed as to avoid the necessity of coordinated pouring schedule and intermediate grouting of joints. This 'self-standing' condition implies that the excentricity of the vertical deadweight load acting on each horizontal section of an isolated monolith is everywhere contained within $1 / 6$ of the width of the section. In order to take maximum advantage, under the design load, of the dead-weight pre-stressing thus generated, it is necessary that this excentricity be directed downstream in the upper part of the cantilever and upstream in the lower part. The excentricity diagram under the design load is qualitatively reversed (i.e. directed upstream of the section centre in the upper part of the cantilever and downstream in the lower part), and to avoid in-service tensile vertical stresses it should also remain contained in either zone within $1 / 6$ of the local section width. These two constraints are inherently embodied in the basic model herein adopted, so that it is unnecessary to check their compliance 'a posteriori'. The 'pilot' element of the design-load splitting becomes now the crown cantilever (In the traditional RITTER approach the pilot elements are the arches, whose radius is pre-defined, and the load fraction not absorbed by the arches must be taken up by the cantilevers. Therefore there is no 'a priori' assurance that vertical tensile stresses are avoided, but one has to check this condition 'a posteriori' and to accept or modify the design on the basis of the check results.), which is assumed to take up just the load fraction it can carry without tensile stresses; the remaining fraction taken up by the arches is then determined by difference with the design load, and the crown radii of curvature are accordingly defined. In the simplified presentation of our linear model (next §3) the following assumptions are made: -a symmetrical configuration is assumed, and only the equilibrium of the central cantilever is explicitly formulated,

-the design load is assumed to consist of horizontal actions (external pressures on the upstream face); uplift is disregarded,

-the mutual actions exchanged between arches and central cantilever are likewise assumed to be horizontal; torsional stiffness is neglected, -the foundation compliance is represented through the VOGT coefficients, -thermal loads are not included in the formal load-splitting (they can be taken account of in the final stress checks); dynamic actions are replaced by pseudostatic ones according to the WESTERGAARD theory. 
Extensive prior experience of the Authors in design (or in back-analysis of inservice behaviour) of large arch dams gave empirical evidence that these approximations do not significantly distort the structural modelling. Besides, the uncertainties about the input data and the necessarily sketchy character of the design load are not logically consistent - always in the Authors' opinion - with the use of very sophisticated models.

\section{Basic equations, parameter fields of variation, search strategy}

The following presentation implies reference to a 'reduced scale model' of the dam in which the actual height $H$ is brought down to $1 m$ and the specific weights of water and concrete are increased in the ratio $H / 1$ in order to preserve the magnitude of pressures and stresses. Symbol $\zeta$ denotes the ratio $z / H$, where the vertical coordinate $z$ is measured downwards from the dam crest, and $H$ is the actual height of the dam. Three functions of $\zeta$ define the geometry of the dam: the profile of the central cantilever axis, $\eta(\zeta)$, the law of variation of the thickness with elevation, $\lambda(\zeta)$, and the law of variation of the crown radii with elevation, $R(\zeta)$. Constant-thickness arches are assumed for simplicity. All integrals are definite integrals between limits 0 and $\zeta . E_{c}, E_{r}$ are the Young moduli of concrete and foundation bedrock, respectively.

3.1 - The choice of a function $e(b, \zeta)$ gives the parametric equation of the relative excentricity $f(\zeta)$ along the central monolith under dead-weight:

$$
\begin{aligned}
& f(\zeta)=e(b, \zeta) ; \text { constraints: } e(\zeta=0)=0 \quad, \quad e(\zeta=b)=0 \quad, \quad 0 \leq e(b, \zeta) \leq 1 / 6 \quad \text { for } \\
& 0 \leq \zeta \leq b, \quad-1 / 6 \leq e(b, \zeta) \leq 0 \text { for } b \leq \zeta \leq 1 \quad \text { (b is our first parameter) }
\end{aligned}
$$

Under design load the relative excentricity $f_{L}(\zeta)$ along the central monolith is defined by another suitable function $e_{L}\left(b_{L}, \zeta\right)$, where $b_{L}$ are either equal or distinct from $b: f_{L}(\zeta)=-e_{L}\left(b_{L}, \zeta\right)$, with constraints:

$$
\begin{gathered}
e_{L}(\zeta=0)=0 \quad, \quad e_{L}\left(\zeta=b_{L}\right)=0 \quad, \quad 0 \leq e_{L}\left(b_{L}, \zeta\right) \leq 1 / 6 \quad \text { for } \quad 0 \leq \zeta \leq b_{L},-1 / 6 \leq \\
e_{L}\left(b_{L}, \zeta\right) \leq 0 \quad \text { for } b_{L} \leq \zeta \leq 1 \quad\left(b_{L} \text { is our second parameter }\right)
\end{gathered}
$$

The choice of a suitable function $T\left(\Lambda_{0}, \alpha, \zeta\right)$ gives the parametric equation of the section thickness $\lambda(\zeta)$ along the central monolith:

$$
\begin{aligned}
& \lambda(\zeta)=T\left(\Lambda_{0}, \alpha, \zeta\right) \quad \text { where } T(\zeta>0)>T(\zeta=0) \quad\left(\Lambda_{0} \text { and } \alpha\right. \text { are our third and fourth } \\
& \quad \text { parameters })
\end{aligned}
$$

Fig. 1 shows the relative excentricity $f(\zeta)$ as a function of parameter $b$.

The shape of the centerline of crown cantilever (coordinate $y$ being positive towards upstream, and $y=0$ for $\zeta=0$ ) is denoted by:

$y=\eta(\zeta)$; then $\eta(\zeta)$ is to be determined by condition (1). Defining the integral function of $\zeta$ :

$$
\Lambda(\zeta)=\int \lambda(\xi) \cdot d \xi
$$

condition (1) yields the following equation for $\eta(\zeta)$ :

$$
\eta(\zeta)=\int f(\xi) \cdot \lambda^{2}(\xi) \cdot \mathrm{d} \xi / \Lambda(\xi)+f(\zeta) \cdot \lambda(\zeta)
$$

from which $\eta(\zeta)$ is seen to be a parametric function of $b, \Lambda, \lambda$. The dead-weight bending moment $M_{b}(\zeta)$ on a generic horizontal section is then a function of $\eta$, $\lambda, b$ (with $\gamma_{\mathrm{c}}=$ unit weight of concrete): 


$$
M_{b}(\zeta)=\gamma_{c} \cdot[\eta(\zeta) \cdot \Lambda(\zeta)-W(\zeta)]_{b} \quad, \quad \text { where } \quad W(\zeta)=\int \lambda(\xi) \cdot \eta(\xi) \cdot d \xi
$$

Under design load this bending moment must be offset and excentricities of opposite sign, with parameter $b$ changed to $b_{L}$, must be generated; thus the fullload bending moment $M_{L}(\zeta)$ must be:

$$
M_{L}(\zeta)=-M_{b}(\zeta)-M_{b L}(\zeta), \text { a function of } b, b_{L}, \eta, \Lambda, W
$$

From this distribution of bending moments the horizontal pressure carried by the central cantilever at full load, $p_{m}(\zeta)$, can be obtained:

$$
p_{m}(\zeta)=\mathrm{d}^{2} M_{L}(\zeta) / \mathrm{d} \zeta^{2}
$$

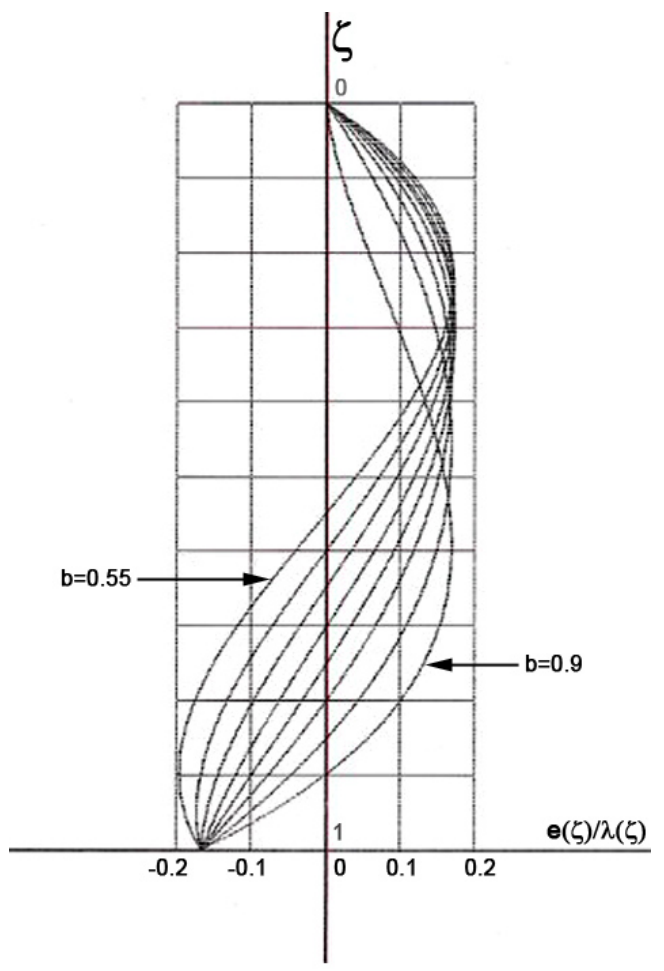

Figure 1: Excentricities.

The full-load elastica $\delta(\zeta)$ is then obtained by double integration of (7):

$$
\begin{gathered}
\phi(\zeta)=-12 . \int M_{L}(\xi) \cdot \mathrm{d} \xi /\left[E_{c} \cdot \lambda^{3}(\xi)\right]+\phi(\zeta=0) \\
\delta(\zeta)=-\int \phi(\zeta) \cdot \mathrm{d} \xi+\delta(\zeta=0)
\end{gathered}
$$

The two integration constants $\phi(\zeta=0)$ and $\delta(\zeta=0)$ are determined by the conditions of compliance of the rotation and, respectively, of the radial displacement at the interface central cantilever/foundation $(\zeta=1)$. Now the generic arch, of crown radius $R(\zeta)$ and thickness $\lambda(\zeta)$, undergoing at crown the same radial displacements $\delta(\zeta)$ as those of the central cantilever, reacts with a pressure $p_{a}(\zeta)$ given approximately by: 


$$
p_{a}(\zeta)=p_{\text {ext }}(\zeta)-p_{m}(\zeta) \approx(8 / 15) \cdot E_{c} \cdot \delta(\zeta) \cdot \lambda(\zeta) / R^{2}
$$

where $p_{\text {ext }}(\zeta)$ is the external pressure; Fig. 2 shows the standard distribution adopted (hydrostatic load plus an excess pressure distribution covering 'average' extra loads such as seismic inertial forces). From (8), (10) and (11) the last unknown, $R(\zeta)$, is obtained. Checks on horizontal (arch) stresses follow.

3.2 - Parameter fields of variation: The intervals of values within which the basic parameters are made to vary are chosen by the designer on the basis of his/her experience. As a rule of thumb the following interval limits can be suggested: $0.55<b<0.8 ; 0.05<\Lambda_{0}<0.15 ; 0.8<\alpha<1.2$.

3.3 - Strategy of search: A discrete scanning of the presumed fields of variation of the basic parameters is established, giving the upper and lower bounds for each, as well as the desired number of sampling steps. Then for each possible combination of these parameters' values the computations indicated by formulas (1) to (11) under $\S 3.1$ are performed. For each parameter combination checks are moreover performed on arch stresses and the solutions exceeding the stress limits imposed as input data are discarded. Those results that are manifestly un-physical are rejected, and for each of the remaining ones the cost (or simply the concrete volume) is computed. The smaller of the latter figures, together with the quantities defining the relevant dam geometry, are retained as the 'optimal' solution. See further on Fig. 3 for details.

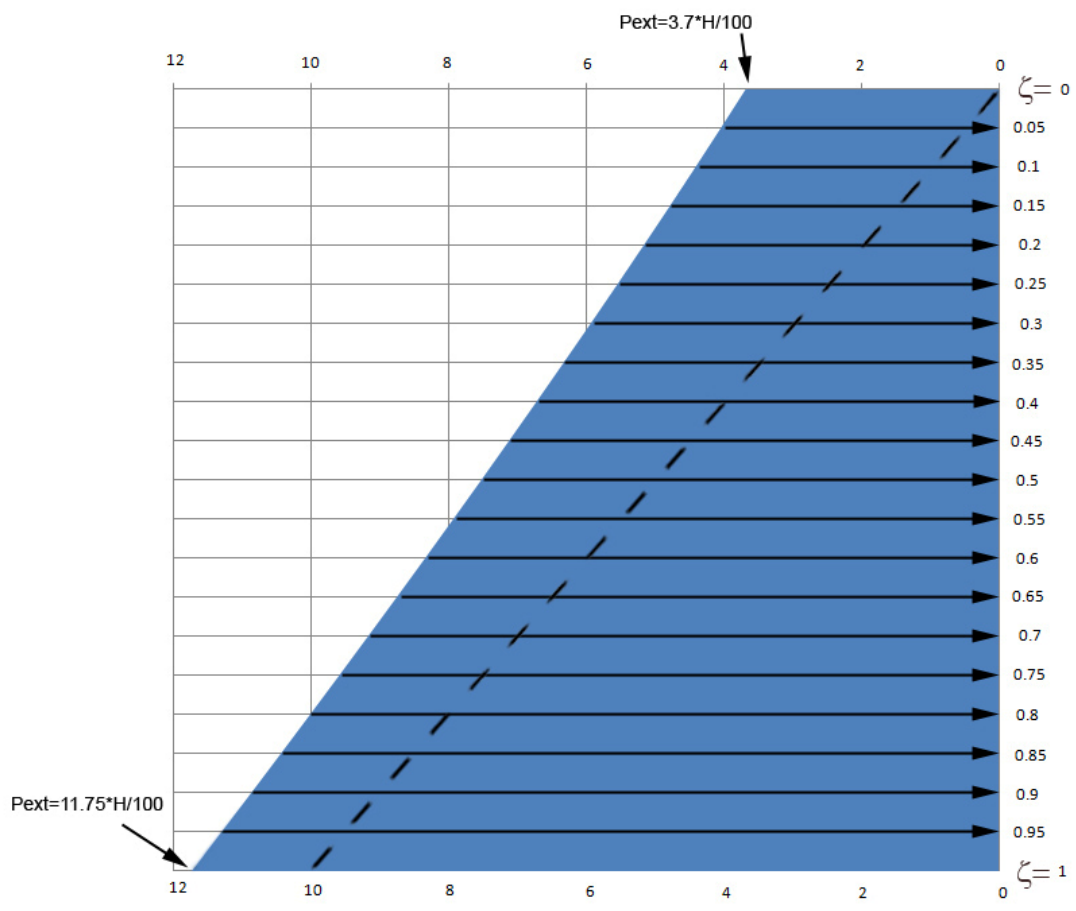

Figure 2: Standard load: external pressures $\left(\mathrm{kg} / \mathrm{cm}^{2}\right)$ (dashed line $=$ hydrostatic load). 


\section{Code structure and an example of application}

Figure 3 shows the successive steps of the computational procedure, evidencing that sometimes two 'optimal' shapes with the same volume can be obtained, since the excentricity curves for dead-weight and for full load are interchangeable (except for the sign). The next Fig. 4 shows the input form, while Fig. 5 exemplifies the graphical presentation of the code output showing the results obtained for a dam $100 \mathrm{~m}$ high in a valley whose transverse section is depicted in the same figure. A few supplementary comments are in order:

-the 'optimal' shapes are quite sensitive to the limits imposed on concrete stresses; the designer may use this kind of information to achieve the best technical/economical balance between volume and cement content, -the shapes obtained look adequate as a starting point for the dam final design.

\section{Conclusions}

5.1 - In the Authors' opinion the above outlined approach suggests that the degrees of freedom which it is advisable to adopt in the shape optimisation procedure of an arch dam can be reduced in practice - by a judicious choice of basic model and independent variables- to a very small number, probably as low as only three or four. Past research based on similar lines, though less stringently pursued, has indeed shown that clever optimization procedures based on only two to four degrees of freedom can yield shapes (and volumes) fairly comparable to those obtained by the use of much more complicated formulations, based on a high number of degrees of freedom for the shape definition and on F.E. structural modeling. In the above-abridged presentation the degrees of freedom are essentially three (or four if one considers that $b$ can vary between the conditions of 'dead-weight on free-standing monoliths' and 'design load'):

$$
a_{\varepsilon} \quad A_{0} \quad b .
$$

The basic model requires only enchained computations, avoiding the solution of huge systems of equations; thus computation times are kept small.

5.2 - From a more general point of view, the Authors think that in order to take into account the ample diversity of loads acting on the dam throughout its lifespan the definition of the optimization 'objective function' should be given more careful, critical thought. Very sketchily, in the Authors' opinion an ideal 'rational' approach could be imagined more or less as follows. The external load scenarios should be defined in detail, assigning an estimate of the probability $P_{L}$ of occurrence of each scenario during the dam's lifetime. Then for each of these load sets the 'vulnerability', or the probability of failure of the dam, $V_{P}$ (as an implicit function of the shape parameters) should be evaluated; lastly, the $\operatorname{cost} C_{F}$ of the consequences of each case of 'failure' should also be evaluated. Maintenance costs, $C_{m}$, and operating costs, $C_{e}$, should also be included. The total cost to be minimized, $T C$, could then be expressed as:

$$
T C=\sum\left(C_{c}+C_{e}+C_{m}\right)+\sum_{L} P_{L} \cdot V_{P} \cdot C_{F}
$$




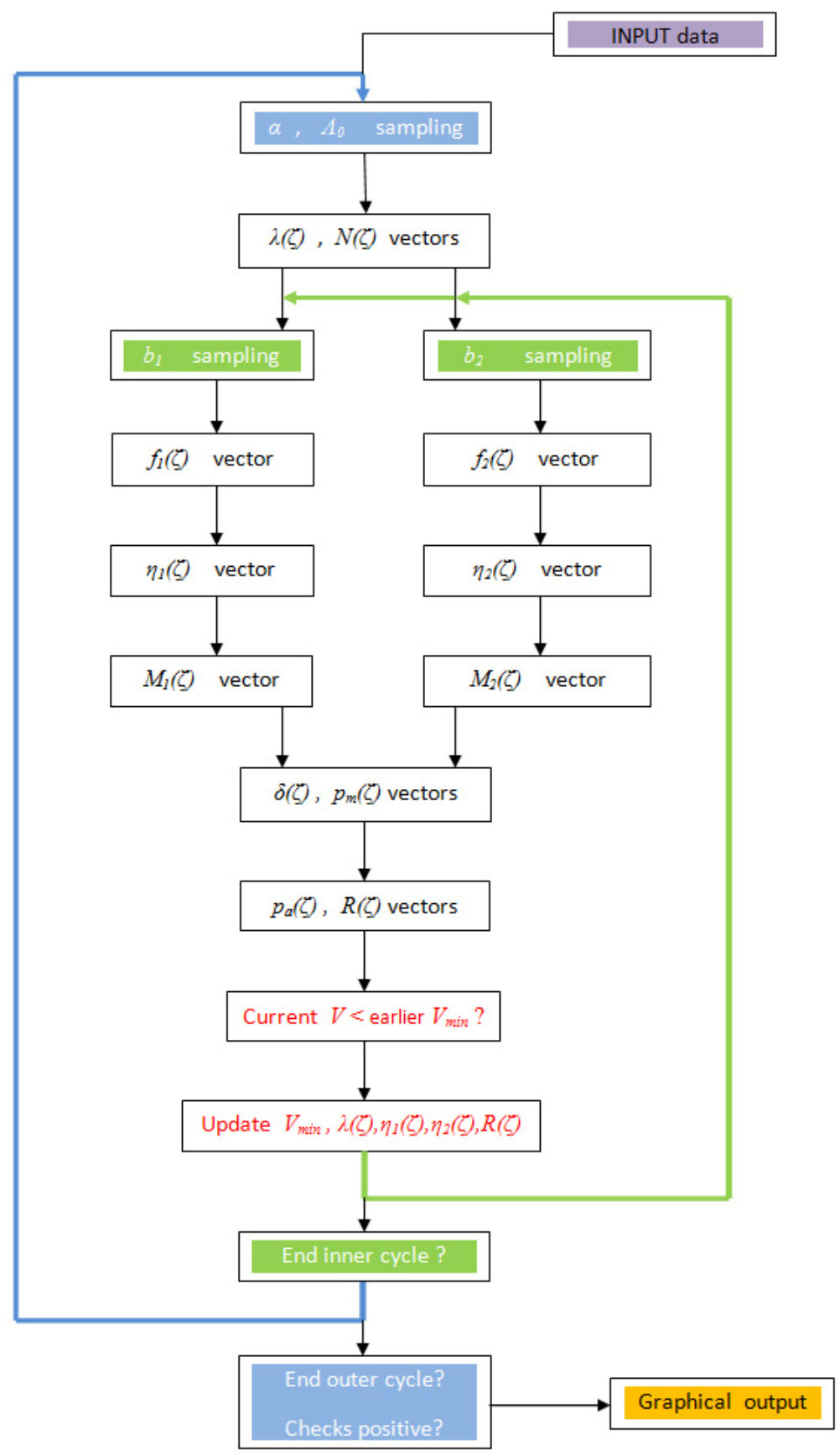

Figure 3: $\quad$ Flow chart of optimisation procedure. 


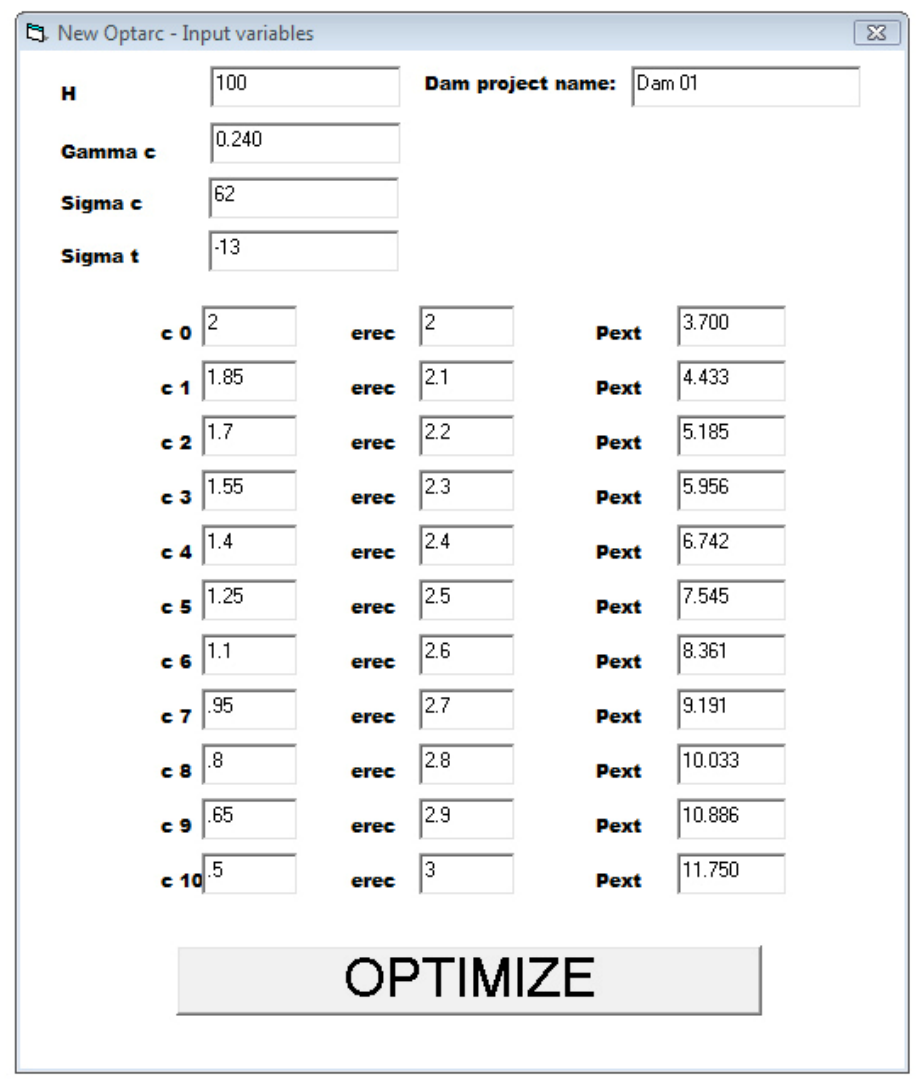

Figure 4: Input form for example of Fig. 5. The values $\mathrm{c}(\mathrm{I})$ stand for the ratio $\mathrm{C}(\mathrm{z}) / \mathrm{H}$ where $\mathrm{C}(\mathrm{z})$ is the valley chord at $\mathrm{z}$ meters under crest; $\mathrm{z}=$ $\mathrm{I} * \mathrm{H} / 10$. erec $=\mathrm{Ec} / \mathrm{Er}$.

[All time-dependent costs should of course be actualised to a convenient date.] Such an objective function would be preferable to the above simpler one $(\S \mathbf{1})$, and its adoption would make reasonable the use of sophisticated models; but, alas! the outstanding difficulties of putting into practice such a 'rational' approach are all too evident.

5.3 - In the end, it goes without saying that any algorithmic 'optimisation' procedure should be followed by an 'expert' refining of the design in order to bring it into line with actual circumstances and with good practice. In this connection it is necessary that the designer's experience and intuition complement the results of the chosen algorithmic tool if an engineeringly 'safe and sound' optimal design is to be achieved. 
음

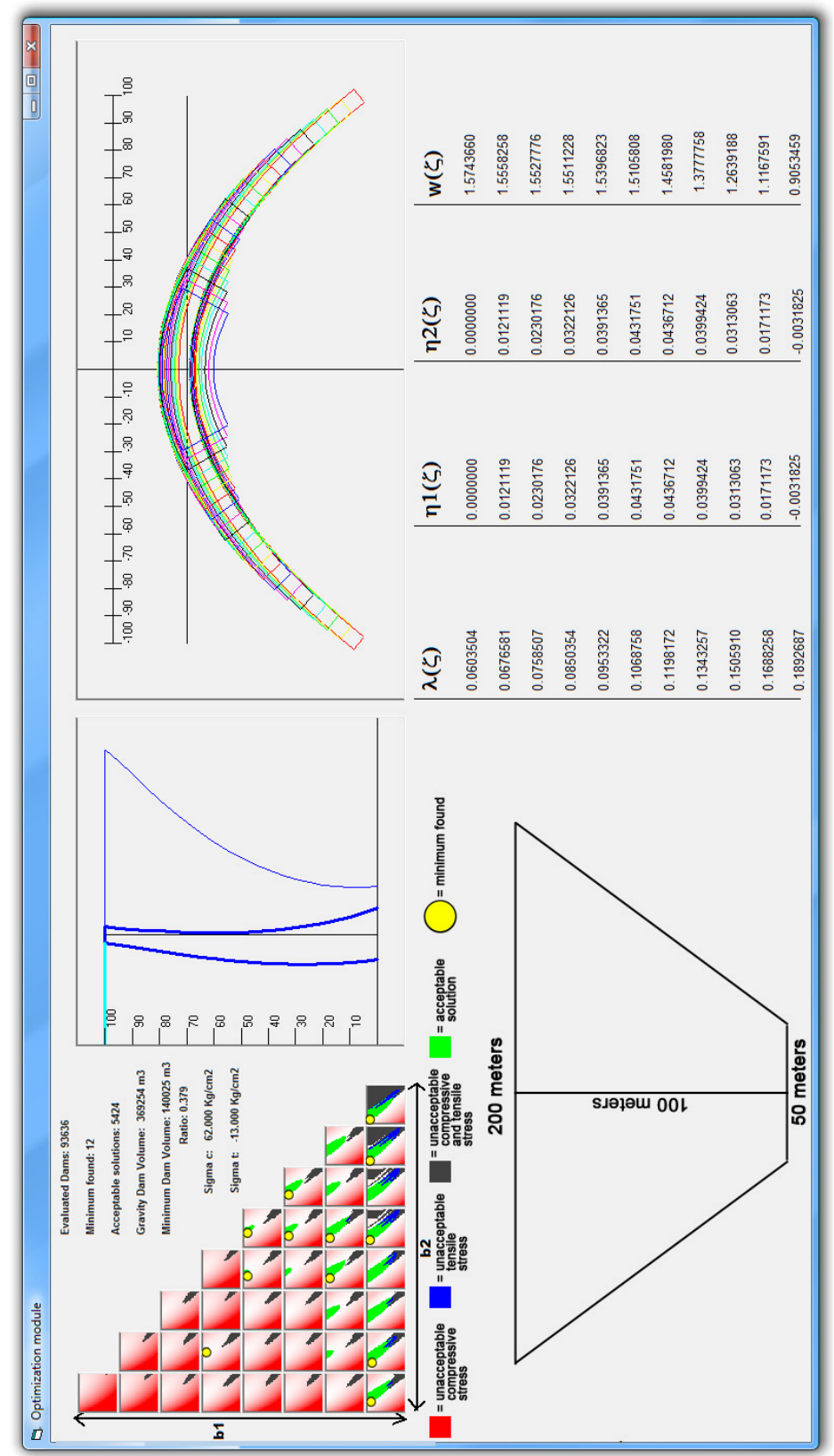

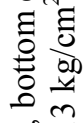

छก

융

$\|$

흥

$\frac{1}{0}$

氙

0

要

츨

$>=$

तี

옹

츤

त

$\Xi 0$

की हี

$\exists \cdot \bar{c}$

घ

으

寻 $\frac{0}{\sqrt{n}}$

$\pi$ के

ᄒํํㄹ

司

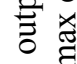

त)

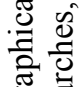

तี

4

중

ब సี

छี

떵ํํ

$\underset{\sim}{\ddot{0}}$ 


\section{References}

[1] Zhu Bofang, Optimum Design of Arch Dams, Dam Engineering, Vol. I, Issue 2, 1990

[2] Zhu Bofang, Rao Bin, Jia Jinsheng, Li Yisheng, Shape Optimisation of Arch Dams for Static and Dynamic Loads, Proceedings of the International Symposium on Practice and Theory of Arch Dams - Hohai University Press, Nanjing (China), 1992

[3] M. Fanelli, Optimal Proportioning of Arch-Gravity Dams: the Automatic Generation of a Simplified Starting Geometry, Dam Engineering, Vol. III, Issue 2, 1992

[4] M. Fanelli, A. Fanelli, P. Salvaneschi, A Neural Network Approach to the Definition of Near Optimal Arch Dam Shape, Dam Engineering, Vol. I, Issue 2, 1992

[5] Orsola Brasi, Personal communication, January 2000, Metodo approssimato per l'ottimizzazione di forma delle dighe ad arco-gravità (An approximated Method for the Shape Optimisation of Arch-Gravity Dams; a thesis discussed at the Polytechnic School of Milano (referees M. \& A. Fanelli)

[6] Stoyan Grigorov, Stefan Tasev and Anton Tzenkov, Extended Optimisation of Arch Dams, Tenth Bulgarian Conference on Theoretical Mechanics, Varna (Bulgaria), 2005.

[7] Jiazheng Pan and Jing He, Shape Optimisation of Arch Dams (Chapt. 7). Large Dams in China, China Water Power Press, pages 443-482, 2000. 